\title{
FOLFOX-7 Regimen
}

National Cancer Institute

\section{Source}

National Cancer Institute. FOLFOX-7 Regimen. NCI Thesaurus. Code C63592.

A reg imen consisting of leucovorin, infusional fluorouracil and oxaliplatin used for the treatment of advanced-stage and metastatic colorectal cancer. This version differs from the orig inal FOLFOX (FOLFOX4) regimen with regards to both the doses and the administration of the three-drug combination. 